Research Article

\title{
Monotonicities in Orlicz Spaces Equipped with Mazur-Orlicz F-Norm
}

\author{
Xinran Bai, ${ }^{1}$ Yunan Cui ${ }^{10},{ }^{1}$ and Joanna Kończak ${ }^{2}$ \\ ${ }^{1}$ Department of Mathematics, Harbin University of Science and Technology, Harbin 150080, China \\ ${ }^{2}$ Faculty of Mathematics and Computer Science, Adam Mickiewicz University in Poznań, ul. Umultowska 87, 61-614 Poznań, Poland
}

Correspondence should be addressed to Yunan Cui; cuiya@hrbust.edu.cn

Received 25 December 2019; Revised 29 April 2020; Accepted 4 May 2020; Published 31 May 2020

Academic Editor: Gen Q. Xu

Copyright (C) 2020 Xinran Bai et al. This is an open access article distributed under the Creative Commons Attribution License, which permits unrestricted use, distribution, and reproduction in any medium, provided the original work is properly cited.

\begin{abstract}
Some basic properties in Orlicz spaces and Orlicz sequence spaces that are generated by monotone function equipped with the Mazur-Orlicz F-norm are studied in this paper. We give some relationships between the modulus and the Mazur-Orlicz $F$-norm. We obtain an interesting result that the norm of an element in line segments is formed by two elements on the unit sphere less than or equal to 1 if and only if that the monotone function is a convex function. The criterion that Orlicz spaces and Orlicz sequence spaces that are generated by monotone function equipped with the Mazur-Orlicz $F$-norm are strictly monotone or lower locally uniform monotone is presented.
\end{abstract}

\section{Introduction}

Geometry of Banach space has important applications in the control theory, fixed-point theory, ergodic theory, probability theory, and vector analytic function theory. Recall that the monotonicity properties are restrictions of appropriate rotundity properties to the set of couples of comparable elements in the positive cone of a Banach lattice (see [1-5]). Consequently, then in many cases, good convex properties can be replaced successfully by respective monotonicity properties (see [6-8]). It is well known that monotonicity properties (strict and uniform monotonicity) play an analogous role in the best dominated approximation problems in Banach lattices as the respective rotundity properties (strict and uniform rotundity) in the best approximation problems in Banach spaces (see $[9,10])$. Moreover, monotonicity properties are applicable in the ergodic theory, since they provide a tool for estimating a norm (see [11-13]). In this paper, based on the paper in 2019 by Cui et al., after removing the continuous condition of generating function (see [6]), we found some conclusions completely different from those in the Banach spaces we studied in the past, which provided us with a new understanding and enlightenment for studying geometric properties of seminormed linear spaces in the future.

\section{General Auxiliary and New Results}

Definition 1. Let $(G, \Sigma, \mu)$ be a $\sigma$-finite measure space and $L^{0}(\mu)$ be the space of all (equivalence) classes of $\Sigma$-measurable real-valued funcions defined on $G$. A function $\Phi:(-\infty, \infty)$ $\longrightarrow[0, \infty]$ is called an Orlicz function if $\Phi(u)>0$ for all $u \neq$ $0 \lim _{u \rightarrow 0+0} \Phi(u)=0$, and even $\Phi$ is the nondecreasing function and $\lim _{u \rightarrow \infty} \Phi(u)=\infty$. Any Orlicz function $\Phi$ determines a functional $I_{\Phi}: L^{0}(\mu) \longrightarrow[0, \infty]$ defined by the formula $I_{\Phi}(f)=$ $\int_{G} \Phi(|f(t)|) d \mu(t)$ and called the modular. The order ideal

$$
L^{\Phi}(\mu)=\left\{f \in L^{0}(\mu): I_{\Phi}(r f)<\infty \text { for some } r>0\right\}
$$

in $L^{0}(\mu)$ is called an Orlicz space.

Let us define a lattice ideal $E^{\Phi}(\mu)$ in $L^{\Phi}(\mu)$ as

$$
E^{\Phi}(\mu)=\left\{f \in L^{0}(\mu): I_{\Phi}(r f)<\infty \text { for all } r>0\right\} .
$$

The space $L^{\Phi}(\mu)$ is linear with respect to the following lattice $F$-norm, called the Mazur-Orlicz F-norm (see [6]): 


$$
\|f\|_{F}=\inf \left\{\lambda>0: I_{\Phi}(f / \lambda) \leq \lambda\right\} .
$$

Note that if $\Phi$ is a convex function, then $L^{\Phi}(\mu)$, endowed with the equivalent lattice norm (called the Luxemburg norm) $\|f\|_{\Phi}=\inf \left\{\lambda>0: I_{\Phi}(f / \lambda) \leq 1\right\}$, becomes a Banach lattice. If $\Phi$ is right continuous and $X$ is a Banach space, increasing function on $[0,+\infty]$ such that $\Phi(0)=0$. Define

$L_{\Phi}(\mu, X)=\left\{f \in L_{0}(\mu, X): \int_{G} \Phi\left(\frac{\|f(x)\|}{\rho}\right) d \mu(x)<\infty\right.$ for some $\left.\rho>0\right\}$.

The properties of $\Phi$ yield that if for $f \in L_{\Phi}(\mu, X)$, we put

$$
\|f\|_{\Phi}=\inf \left\{\rho>0: \int_{G} \Phi\left(\frac{\|f(x)\|}{\rho}\right) d \mu(x)<\rho\right\}
$$

then $\|\cdot\|_{\Phi}$ satisfies the axioms of a $\Delta$-norm in $L_{\Phi}(\mu, X)$ (see [14]). Moreover, $\|\cdot\|_{\Phi}$ is equivalent to an $F$-norm under which $L_{\Phi}(\mu, X)$ is complete, whence $\left(L_{\Phi}(\mu, X),\|\cdot\|_{\Phi}\right)$ is an F-space.

In the case of the counting measure space, the space $L^{\Phi}$ $(\mu)=l^{\Phi}$ is always nontrivial and

$$
l^{\Phi}=\left\{x \in l^{0}: \forall_{\lambda>0} \exists_{n_{\lambda} \in \mathbb{N}} \sum_{n=n_{\lambda}}^{\infty} \Phi(\lambda x(n))<\infty\right\}=: h^{\Phi} .
$$

Remark 2. If $\Phi$ is not right continuous at zero, then $\lim _{u \rightarrow 0+0}$ $\Phi(u)=a>0$. If we take $x_{n}(t)=(1 / n) \chi_{G}(t)$, then $x_{n}(t)$ is convergent to 0 uniformly, but $I_{\Phi}\left(x_{n}\right)=\int_{G} \Phi\left((1 / n) \chi_{G}(t)\right) d$ $t=\Phi(1 / n) m(G) \geq a m(G)$ for all $n \in N$. This means that the case $\lim _{u \rightarrow 0+0} \Phi(u)>0$. We do not even have the fact that the uniform convergence of the sequence $\left\{x_{n}\right\}$ implies its modular convergence.

Lemma 3. $L^{\Phi}(\mu)$ is a linear space.

Proof. Obviously, if $\Phi$ is a monotone increasing function, then for all $u, v \in R$ and $\alpha \in(0,1)$ we have

$$
\Phi(\alpha u+(1-\alpha) v) \leq \Phi(u)+\Phi(v)
$$

Take any $f, g \in L^{\Phi}(\mu)$, by the definition of the $L^{\Phi}(\mu)$; there exists $\lambda_{1}, \lambda_{2}>0$ such that

$$
\int_{G} \Phi\left(\lambda_{1} f(x)\right) d x<\infty, \int_{G} \Phi\left(\lambda_{2} f(x)\right) d x<\infty
$$

We choose $\lambda_{0}=\min \left\{\lambda_{1}, \lambda_{2}\right\}$, that is,

$$
\int_{G} \Phi\left(\lambda_{0} f(x)\right) d x<\infty, \int_{G} \Phi\left(\lambda_{0} f(x)\right) d x<\infty
$$

Assume now that $a \neq 0$ or $b \neq 0$, and we conclude that

$$
\begin{aligned}
\int_{G} \Phi\left(\frac{\lambda_{0}}{|a|+|b|}(a f(x)+b g(x))\right) \\
\cdot d x \leq \int_{G} \Phi\left(\frac{\lambda_{0}}{|a|+|b|}(|a||f(x)|+|b||g(x)|)\right) \\
\cdot d x \leq \int_{G} \Phi\left(\lambda_{0} f(x)\right) d x+\int_{G} \Phi\left(\lambda_{0} g(x)\right) d x<\infty .
\end{aligned}
$$

Hence, $L^{\Phi}(\mu)$ is a linear space.

Definition 4 (see [6]). An F-normed Orlicz space $\left(L^{\Phi}(\mu)\right.$, $\|\cdot\|_{F}$ ) is said to be strictly monotone (we write $L^{\Phi}(\mu) \in(S M)$ ) if for any $x, y \in L^{\Phi}(\mu)$ such that $0 \leq y \leq x$, we have $\|y\|_{F}<$ $\|x\|_{F}$ whenever $y \neq x \quad$ (or equivalently $\|x-y\|_{E}<\|x\|_{E}$ whennever $y \neq 0$ ).

Definition 5 (see [6]). An F-normed Orlicz space $\left(L^{\Phi}(\mu),\|\cdot\|_{F}\right)$ is said to be lower locally uniformly monotone (we write $\left.^{\Phi}(\mu) \in(L L U M)\right)$ if for anyx $\in L^{\Phi}(\mu)$ and $\left(x_{n}\right)_{n=1}^{\infty}$ $\operatorname{inL}^{\Phi}(\mu)$ such that $0 \leq x_{n} \leq x$ for alln $\in \mathbb{N}$ and $\left\|x_{n}\right\|_{F} \longrightarrow$ $\|x\|_{F}$ asn $\longrightarrow \infty$, the condition $\left\|x-x_{n}\right\|_{F} \longrightarrow 0$ as $n \longrightarrow \infty$ holds.

Definition 6. We say that $\Phi$ satisfies $\Delta_{2}$-condition $\left(\Phi \in \Delta_{2}\right.$ for short) whenever there are constants $K>0$ and $u_{0}>0$ such that (see [15])

$$
\Phi(2 u) \leq K \Phi(u)\left(u \geq u_{0}\right)
$$

Definition 7. We say that $\Phi$ satisfies $\Delta_{l}$-condition $\left(\Phi \in \Delta_{l}\right.$ for short) whenever there are constants $K_{1}>0$ and $u_{1}>0$ such that

$$
\Phi(l u) \leq K_{1} \Phi(u)\left(u \geq u_{1}\right)
$$

Lemma 8. $\Phi \in \Delta_{2}$ if and only if $\Phi \in \Delta_{l}$ for all $l>1$.

Proof. Sufficiency: given that $l>1$, choose an integer $n_{0}$ such that $l^{n_{0}}>2$. Then, by (12), we have

$$
\Phi(2 u) \leq \Phi\left(l^{n_{0}} u\right)<K_{1}^{n_{0}} \Phi(u)\left(u \geq u_{1}\right)
$$

Necessity: now choose an integer $n_{1}$ such that $2^{n_{1}}>l$. Then, by (11) we get

$$
\Phi(l u) \leq \Phi\left(2^{n_{1}} u\right) \leq K^{n_{1}} \Phi(u)\left(u \geq u_{0}\right)
$$

which ends the proof.

Definition 9. We say that $\Phi$ satisfies $\delta_{2}$-condition $\left(\Phi \in \delta_{2}\right.$ for short) whenever there are constants $k_{1}>0$ and $u_{2}>0$ such that

$$
\Phi(2 u) \leq k_{1} \Phi(u)\left(|u| \leq u_{2}\right)
$$

Theorem 10. If $\Phi$ is an Orlicz function such that $\Phi \in \Delta_{2}$, then 
for any $\varepsilon>0$, there exists $K>0$ such that

$$
I_{\Phi}(2 f) \leq K I_{\Phi}(f)+\varepsilon
$$

for all $f \in L^{\Phi}(\mu)$.

Proof. By $\Phi \in \Delta_{2}$, there exists $u_{1}>0$ and $K_{1}>0$ such that $\Phi$ $(2 u) \leq K_{1} \Phi(u) \quad\left(\forall u \geq u_{1}\right)$. Let us assume that $0<u_{0}<u_{1}$ and for any $u \in\left[u_{0}, u_{1}\right]$ and $\Phi\left(u_{0}\right)>0$, we get

$$
\frac{\Phi(2 u)}{\Phi(u)} \leq \frac{\Phi\left(2 u_{1}\right)}{\Phi\left(u_{0}\right)}
$$

We choose $K=\max \left\{K_{1}, \Phi\left(2 u_{1}\right) / \Phi\left(u_{0}\right)\right\}$. We can hence easily get that $\Phi(2 u) \leq K \Phi(u)$ for any $u \geq u_{0}$. By $\lim _{u \rightarrow 0+0} \Phi(u)=0$, for any $\varepsilon>0$, there exists $\delta>0$ such that $\Phi\left(2 u_{0}\right) m(G)<\varepsilon$, whenever $u_{0}<\delta$. Defining $G_{n}=\{x \in G: \mid$ $\left.f(x) \mid \leq u_{0}\right\}$, we obtain

$$
\begin{aligned}
I_{\Phi}(2 f)= & \int_{G_{n}} \Phi(2 f(x)) d x+\int_{G \backslash G_{n}} \Phi(2 f(x)) \\
& \cdot d x \leq \Phi\left(2 u_{0}\right) m(G)+K \int_{G} \Phi(f(x)) \\
& \cdot d x \leq K I_{\Phi}(f)+\varepsilon .
\end{aligned}
$$

Theorem 11. For any $f \in L^{\Phi}(\mu), I_{\Phi}\left(f /\|f\|_{F}\right)=\|f\|_{F}$ if and only ifФis right continuous and $\Phi \in \Delta_{2}$.

Proof. Sufficiency: for any $f \in L^{\Phi}(\mu) \backslash\{0\}$ the function $F(\lambda)=I_{\Phi}\left(\lambda f /\|f\|_{F}\right)$ is right continuous on the interval $(0, \infty)$. By the definition of $\|f\|_{F}$, we have $F(1) \leq$ $\|f\|_{F}$ and for any $\lambda>1$, we have $F(\lambda)>\|f\|_{F}$. Using $\lim _{\lambda \rightarrow 1+0} \Phi(\lambda f(x)) \longrightarrow \Phi(f(x))$ and the Lebesgue dominated convergence theorem, we obtain

$$
\|f\|_{F} \geq I_{\Phi}\left(\frac{f}{\|f\|_{F}}\right)=\lim _{\lambda \rightarrow 1^{+}} \int_{G} \Phi\left(\frac{\lambda f(t)}{\|f\|_{F}}\right) d t
$$

which finishes the proof of the implication $I_{\Phi}\left(f /\|f\|_{F}\right)$ $=\|f\|_{F}$.

Necessity: assume that $\Phi$ does not satisfy the $\Delta_{2}$-condition. This yields that there exists a strictly increasing sequence $\left\{u_{n}\right\}_{n=1}^{\infty}$ of position numbers such that $\Phi\left(u_{1}\right) m(G) \geq 1$ and

$$
\Phi\left(\left(1+\frac{1}{n}\right) u_{n}\right)>2^{n} \Phi\left(u_{n}\right)(n=1,2,3, \cdots) .
$$

Next, choose a sequence $\left\{G_{n}\right\}_{n=1}^{\infty}$ of pairwise disjoint sets in $G$ such that $m\left(G_{n}\right)=1 / 2^{n} \Phi\left(u_{n}\right)(n=1,2,3, \cdots)$. Defining $x=\sum_{n=1}^{\infty} u_{n} \chi_{G_{n}}$, we have

$$
I_{\Phi}(x)=\sum_{n=1}^{\infty} \int_{G_{n}} \Phi(x(t)) d t=\sum_{n=1}^{\infty} \Phi\left(u_{n}\right) m\left(G_{n}\right)=\sum_{n=1}^{\infty} \frac{1}{2^{n}}=1 .
$$

On the other hand, we have that for any $\lambda \in(0,1)$, there exists $n_{1} \in N$ such that $1+(1 / n)<\lambda$ for any $n \geq n_{1}$. Hence,

$$
\begin{aligned}
I_{\Phi}(\lambda x) & =\int_{G} \Phi(\lambda x(t)) d t=\sum_{n=1}^{\infty} \Phi\left(\lambda u_{n}\right) m\left(G_{n}\right) \\
& \geq \sum_{n=1}^{\infty} \Phi\left(\left(1+\frac{1}{n}\right) u_{n}\right) m\left(G_{n}\right) \\
& \geq \sum_{n=1}^{\infty} 2^{n} \Phi\left(u_{n}\right) m\left(G_{n}\right)=\sum_{n=1}^{\infty} 1=\infty .
\end{aligned}
$$

Consequently, this shows that $\Phi \in \Delta_{2}$ is a necessary condition.

Next, we will prove the necessary of the rightcontinuous function of $\Phi$. If $\Phi$ is not right continuous on $R$, then there exists a point $u_{0} \in(0,+\infty)$ such that $\Phi$ $\left(u_{0}\right)<\lim _{u \rightarrow u_{0}+0} \Phi(u)=b$; that is, for any $\lambda<1$, we get $\Phi\left(u_{0}\right.$ $/ \lambda) \geq b$. Take any $G_{0} \subset G$ with $\Phi\left(u_{0}\right) m\left(G_{0}\right)<1$. Let us take $c>0$ large enough. Since $\lim \Phi(u)=+\infty$, we have

$$
\Phi\left(u_{0}\right) m\left(G_{0}\right)+\Phi(c) m\left(G G_{0}\right)>1
$$

Since $G$ is nonatomic, we can find $G_{2} \subset G \backslash G_{0}$ such that

$$
\Phi\left(u_{0}\right) m\left(G_{0}\right)+\Phi(c) m\left(G_{1}\right)=1-\frac{b-\Phi\left(u_{0}\right)}{2} m\left(G_{0}\right)
$$

Let us define

$$
x=u_{0} \chi_{G_{0}}+c \chi_{G_{1}}
$$

Then, $\quad I_{\Phi}(x)=\Phi\left(u_{0}\right) m\left(G_{0}\right)+\Phi(c) m\left(G_{1}\right)<1$, whence we obtain that $x \in L^{\Phi}(\mu)$ and $\|x\|_{F} \leq 1$. On the other hand, for any $\lambda>1$, we have

$$
\begin{aligned}
I_{\Phi}\left(\frac{x}{\lambda}\right)= & \Phi\left(\frac{u_{0}}{\lambda}\right) m\left(G_{0}\right)+\Phi\left(\frac{c}{\lambda}\right) m\left(G_{1}\right)>b m\left(G_{0}\right) \\
& +\Phi(c) m\left(G_{1}\right)=b m\left(G_{0}\right)+\left(1-\frac{b-\Phi\left(u_{0}\right)}{2}\right) m\left(G_{0}\right) \\
& -\Phi\left(u_{0}\right) m\left(G_{0}\right)=1+\frac{b-\Phi\left(u_{0}\right)}{2} m\left(G_{0}\right)>1
\end{aligned}
$$

which gives that $\|x\|_{F} \geq 1$ yields $\|x\|_{F}=1$, a contradiction, which finishes the proof of necessity.

For convenience, from now on, we write $S\left(L^{\Phi}(\mu)\right)=\{f \in$ $\left.L^{\Phi}(\mu):\|f\|_{F}=1\right\}$. which contradicts the assumption $\|y\|_{F}=$ $\|x\|_{F}$. Therefore, $\|y\|_{F}<\|x\|_{F}$.

Theorem 12. Assume that $x, y \in S\left(L^{\Phi}(\mu)\right)$ and $\alpha \in(0,1)$. Then, $\|\alpha x+(1-\alpha) y\|_{F} \leq 1$ if and only if $\Phi$ is a convex function on $R$. 
Proof. Sufficiency: for any $x, y \in S\left(L^{\Phi}(\mu)\right)$, we have $I_{\Phi}(x) \leq 1$ and $I_{\Phi}(y) \leq 1$. Assume that $z=\alpha x+(1-\alpha) y$. Then, $I_{\Phi}(z) \leq$ $\alpha I_{\Phi}(x)+(1-\alpha) I_{\Phi}(y) \leq 1$. Hence, $\|\alpha x+(1-\alpha) y\|_{F} \leq 1$. This finishes the proof.

Necessity: if $\Phi$ is not a convex function, then there exists $u>v$, such that $\Phi((u+v) / 2)>(1 / 2)(\Phi(u)+\Phi(v))$. Let us choose $G_{1}, G_{2} \subset G$ such that $m\left(G_{1}\right)=m\left(G_{2}\right)$ and $\Phi(u) m\left(G_{1}\right)$ $+\Phi(v) m\left(G_{2}\right) \leq 1$. Take any $c>0$ such that

$$
\Phi(u) m\left(G_{1}\right)+\Phi(v) m\left(G_{2}\right)+\Phi(c) m\left(G_{3}\right)=1
$$

Define

$$
x=u \chi_{G_{1}}+v \chi_{G_{2}}+c \chi_{G_{3}}, y=v \chi_{G_{1}}+u \chi_{G_{2}}+c \chi_{G_{3}}, z=\frac{x+y}{2} .
$$

We obtain

$$
\begin{aligned}
I_{\Phi}(z)= & \Phi\left(\frac{u+v}{2}\right) m\left(G_{1}\right)+\Phi\left(\frac{u+v}{2}\right) m\left(G_{2}\right)+\Phi(c) m\left(G_{3}\right) \\
> & \frac{1}{2}(\Phi(u)+\Phi(v)) m\left(G_{1}\right)+\frac{1}{2}(\Phi(v)+\Phi(u)) m\left(G_{2}\right) \\
& +\Phi(c) m\left(G_{3}\right)=\frac{1}{2}\left(I_{\Phi}(x)+I_{\Phi}(y)\right)=1 .
\end{aligned}
$$

Consequently, $\|x\|_{F}>1$ is a contradiction.

Example 13. Put

$$
\Phi(u)= \begin{cases}u, & |u| \leq 1, \\ 1, & 1 \leq u<2 \\ 2, & 2 \leq u \leq 3 \\ 3 u, & u>3 .\end{cases}
$$

Then, $\Phi$ satisfies the $\Delta_{2}$-condition and $\Phi$ is right continuous. Define

$$
\begin{gathered}
x(t)= \begin{cases}1, & x \in[0,1], \\
0, & x \in(1,2],\end{cases} \\
y(t)= \begin{cases}3, & x \in\left[0, \frac{1}{2}\right], \\
0, & x \in\left(\frac{1}{2}, 2\right] .\end{cases}
\end{gathered}
$$

Then, $y \neq x$ and $I_{\Phi}(x)=\Phi(1) m([0,1])=1$ and $I_{\Phi}(y)=$ $\Phi(3) m([0,1 / 2])=1$. Moreover, $\|y\|_{F}=\|x\|_{F}=1$. Now let us define

$$
z(t)=\frac{1}{3} x+\frac{2}{3} y= \begin{cases}\frac{7}{3}, & x \in\left[0, \frac{1}{2}\right], \\ \frac{1}{3}, & x \in\left(\frac{1}{2}, 1\right], \\ 0, & x \in(1,2] .\end{cases}
$$

Then, $I_{\Phi}(z)=\Phi(7 / 3) m([0,1 / 2])+\Phi(1 / 3) m((1 / 2,1])=2$ $\cdot(1 / 2)+(1 / 3) \cdot(1 / 2)=1+(1 / 6)>1$. This means that $\|z\|_{F}$ $>1$.

Remark 13. This example shows that the norm of an element in a line segment $[x, y]$ consisting of two elements $x$ and $y$ in a unit sphere may be greater than 1. This is essentially different from Banach space $X$. For Banach space $X$, for any $x, y \in S(X)$ and for any $\lambda \in[0,1]$, we get that $[x, y] \subset$ $B(X)=\left\{x \in X:\|x\|_{F} \leq 1\right\}$. Using Theorem 12, ifФis a convex function, we conclude that if $\|x\|_{F} \leq 1,\|y\|_{F} \leq 1$, then $\|\lambda x+(1-\lambda) y\|_{F} \leq 1$.

Theorem 14. The Orlicz space $L^{\Phi}(\mu)$ equipped with the Mazur-Orlicz F-norm $\|\cdot\|_{F}$ is strictly monotone if and only if $\Phi$ is strictly increasing on $R^{+}$and $\Phi$ satisfies the $\Delta_{2}$-condition and $\Phi$ is right continuous.

Proof. Sufficiency: let $0 \leq y \leq x, y \neq x$, then there exists $e_{0} \subset G$ and $m\left(e_{0}\right)>0$ such that $0 \leq y(t)<x(t)\left(t \in e_{0}\right)$. Assuming for the contrary that $\|y\|_{F}=\|x\|_{F}$, we have by Theorem 11,

$$
\begin{aligned}
\|y\|_{F}= & \int_{G \backslash e_{0}} \Phi\left(\frac{y(t)}{\|y\|_{F}}\right) d t+\int_{e_{0}} \Phi\left(\frac{y(t)}{\|y\|_{F}}\right) \\
& \cdot d t<\int_{G \backslash e_{0}} \Phi\left(\frac{x(t)}{\|y\|_{F}}\right) d t+\int_{e_{0}} \Phi\left(\frac{x(t)}{\|y\|_{F}}\right) \\
& \cdot d t=\int_{G \backslash e_{0}} \Phi\left(\frac{x(t)}{\|x\|_{F}}\right) d t+\int_{e_{0}} \Phi\left(\frac{x(t)}{\|x\|_{F}}\right) d t=\|x\|_{F},
\end{aligned}
$$

Necessity: if $\Phi$ is not right continuous on $R$, then there exists a point $u_{0} \in(0,+\infty)$ such that $\Phi\left(u_{0}\right)<\lim _{u \rightarrow u_{0}+0} \Phi(u)=$ $b$; that is, for any $\lambda<1$, we get $\Phi\left(u_{0} / \lambda\right) \geq b$. Take any $G_{0} \subset$ $G$ with $\Phi\left(u_{0}\right) m\left(G_{0}\right)<1$. Let us take $c>0$ large enough. Since $\lim _{u \rightarrow \infty} \Phi(u)=+\infty$, we obtain

$$
\Phi\left(u_{0}\right) m\left(G_{0}\right)+\Phi(c) m\left(G G_{0}\right)>1
$$

Since $G$ is nonatomic, we can find $G_{1} \subset G \backslash\left(G_{0} \cup G_{1}\right)$ such that

$$
\Phi\left(u_{0}\right) m\left(G_{0}\right)+\Phi(c) m\left(G_{1}\right)=1-\frac{b-\Phi\left(u_{0}\right)}{2} m\left(G_{0}\right)
$$

Moreover, take $G_{2} \subset G \backslash\left(G_{0} \cup G_{1}\right)$, where $d$ is a fixed 
positive number such that

$$
\Phi\left(u_{0}\right) m\left(G_{0}\right)+\Phi(c) m\left(G_{1}\right)+\Phi(d) m\left(G_{2}\right)=1
$$

Let us define

$$
x=u_{0} \chi_{G_{0}}+c \chi_{G_{1}}, y=u_{0} \chi_{G_{0}}+c \chi_{G_{1}}+d \chi_{G_{2}} .
$$

Then, $I_{\Phi}(x)=\Phi\left(u_{0}\right) m\left(G_{0}\right)+\Phi(c) m\left(G_{1}\right)<1$, whence we obtain that $x \in L^{\Phi}(\mu)$ and $\|x\|_{F} \leq 1$. On the other hand, we have that for any $\lambda>1$,

$$
\begin{aligned}
I_{\Phi}\left(\frac{x}{\lambda}\right)= & \Phi\left(\frac{u_{0}}{\lambda}\right) m\left(G_{0}\right)+\Phi\left(\frac{c}{\lambda}\right) m\left(G_{1}\right)>b m\left(G_{0}\right) \\
& +\Phi(c) m\left(G_{1}\right)=b m\left(G_{0}\right)+\left(1-\frac{b-\Phi\left(u_{0}\right)}{2}\right) m\left(G_{0}\right) \\
& -\Phi\left(u_{0}\right) m\left(G_{0}\right)=1+\frac{b-\Phi\left(u_{0}\right)}{2} m\left(G_{0}\right)>1
\end{aligned}
$$

which gives that $\|x\|_{F} \geq 1$ yields $\|x\|_{F}=1$. By (36), we obtain $\|x\|_{F}=\|y\|_{F}=1$. Since $0 \leq x \leq y$ and $x \neq y$, this means that the space $\left(L^{\Phi}(\mu),\|\cdot\|_{F}\right)$ is not strictly monotone.

We will prove the necessity of the $\Delta_{2}$-condition. Assume first that $\Phi$ does not satisfy the $\Delta_{2}$-condition. This yields that there exists a strictly increasing sequence $\left(u_{n}\right)_{n=1}^{\infty}$ of positive numbers such that $\Phi\left(u_{1}\right) m(G) \geq 1$ and $\Phi\left((1+(1 / n)) u_{n}\right)>2^{n} \Phi\left(u_{n}\right)$. Let us choose first $G_{1} \subset$ $G$ such that $\Phi\left(u_{1}\right) m\left(G_{1}\right)=1 / 2$. We have $\Phi\left(u_{2}\right) m\left(G G_{1}\right) \geq$ $\Phi\left(u_{1}\right) m\left(G_{1}\right) \geq 1 / 2$. Since $G$ is nonatomic, we can find $G_{2}$ $\subset G \backslash G_{1}$ such that $\Phi\left(u_{2}\right) m\left(G_{2}\right)=1 / 4$. Continuing this procedure by induction, we can find a sequence $\left\{G_{n}\right\}_{n=1}^{\infty}$ of measurable sets such that $G_{n} \subset G \backslash \cup_{k=1}^{n-1} G_{n}$ and $\Phi\left(u_{n}\right)$ $m\left(G_{n}\right)=2^{-n}$, for any $n \in N, n \geq 2$. Let us define

$$
y=\sum_{n=2}^{\infty} u_{n} \chi_{G_{n}}, x=\sum_{n=1}^{\infty} u_{n} \chi_{G_{n}} .
$$

Then, $0 \leq y \leq x, y \neq x$ and $I_{\Phi}(x)=\sum_{n=1}^{\infty} \Phi\left(u_{n}\right) m\left(G_{n}\right)=$ $\sum_{n=1}^{\infty} 2^{-n}=1$, whence we obtain that $x, y \in L^{\Phi}(\mu)$ and $\|x\|_{F}$ $\leq 1$. On the other hand, we have that for any $\lambda \in(0,1)$, there exists $m \in N, m \geq 2$, such that $(1 / \lambda)>1+(1 / n)$ for any $n \geq m$, whence

$$
\begin{aligned}
I_{\Phi}\left(\frac{y}{\lambda}\right) & \geq \sum_{n=m}^{\infty} \Phi\left(\frac{u_{n}}{\lambda}\right) m\left(G_{n}\right) \geq \sum_{n=m}^{\infty} \Phi\left(\left(1+\frac{1}{n}\right) u_{n}\right) m\left(G_{n}\right) \\
& >\sum_{n=m}^{\infty} 2^{n} \Phi\left(u_{n}\right) m\left(G_{n}\right)=\sum_{n=m}^{\infty} 1=\infty
\end{aligned}
$$

which gives that $\|y\|_{F} \geq \lambda$ and by the arbitrariness of $\lambda \epsilon$ $(0,1)$, we get $\|y\|_{F} \geq 1$. The inequalities $0 \leq y \leq x,\|x\|_{F} \leq$ 1 , and $\|y\|_{F} \geq 1$ yield $\|y\|_{F}=\|x\|_{F}$. This equality shows that space $\left(L^{\Phi}(\mu),\|\cdot\|_{F}\right)$ is not strictly monotone.
Now, we will prove the necessity of strict monotonicity of $\Phi$ on $R^{+}$. Assume for the contrary that $\Phi$ is constant on some interval $[a, b] \subset R^{+}$, where $0<a<b<\infty$, so we have $\Phi(a)>0$. Take $A_{1} \in \Sigma$ such that $0<\Phi(a) m\left(A_{1}\right)<1$, and find a positive number $c$ such that $0<\Phi(a) m\left(A_{1}\right)+\Phi(c) m\left(G \backslash A_{1}\right)>1$; using the measure space $\{G, \Sigma, \mu\}$ which is nonatomic, there exists $A_{2} \subset G A_{1}$ such that

$$
\Phi(a) m\left(A_{1}\right)+\Phi(c) m\left(A_{2}\right)=1
$$

Define

$$
x(t)=a \chi_{A_{1}}+c \chi_{A_{2}}, y(t)=b \chi_{A_{1}}+c \chi_{A_{2}}
$$

We obviously have $0 \leq x \leq y, \quad y \neq x, I_{\Phi}(y)=I_{\Phi}(x)=1$ which yields $\|x\|_{F}=\|y\|_{F}=1$; this means that $\|\cdot\|_{F}$ is not strictly monotone.

Theorem 15. $L^{\Phi}(\mu)$ is lower locally uniformly monotone if and only if $\Phi$ is strictly monotone and a right continuous function and $\Phi$ satisfies the $\Delta_{2}$-condition.

Proof. In order to prove the sufficiency of the theorem, assume that $0 \leq x_{n} \leq x \in L^{\Phi}(\mu) \backslash\{0\}$ and $\left\|x_{n}\right\|_{F} \longrightarrow\left\|x_{n}\right\|_{F}$ as $n \longrightarrow \infty$. We need to prove that $\left\|x_{n}-x\right\|_{F} \longrightarrow 0$ as $n \longrightarrow \infty$.

First, we will prove that $x_{n} \longrightarrow x$ in measure. Assuming that $x_{n} \nrightarrow x$ in measure, without loss of generality, then there exists $\varepsilon_{0}>0, \delta_{0}>0$ such that $m\left(\left\{t \in G: x(t)-x_{n}(t) \geq \varepsilon_{0}\right\}\right)$ $\geq \delta_{0}$. Define

$$
G_{n}=\left\{t \in G: x(t)-x_{n}(t) \geq \varepsilon_{0}\right\}
$$

and $z_{n}(t)=x(t) \chi_{G \backslash G_{n}}+\left(x(t)-\varepsilon_{0}\right) \chi_{G_{n}}$ for each $n \in N$. It is clear that $0 \leq x_{n}(t) \leq z_{n}(t) \leq x(t)$ hold. Using condition $\Phi \in$ $\Delta_{2}$, we know that $\int_{G} \Phi(x(t)) d t<\infty$ and then, there exists $D>0$ such that

$$
m(\{t \in G:|x(t)|>D\})<\frac{\delta_{0}}{3} .
$$

Therefore, by conditions (43) and (44), we get that

$$
m\left(\left\{t \in G: x(t) \leq D, z_{n}(t) \leq D, x(t)-z_{n}(t) \geq \varepsilon_{0}\right\}\right) \geq \frac{\delta_{0}}{3} .
$$

Next, we will prove that there exists $\delta_{1}>0$ such that

$$
\Phi\left(u+\varepsilon_{0}\right) \geq \Phi(u)+\delta_{1} 0 \leq u \leq D .
$$

It is obvious that $\Phi\left(u+\varepsilon_{0}\right)>\Phi(u)$, since $\Phi$ is strictly increasing. If (46) does not hold, then $\exists u_{n} \in[0, D]$, such that

$$
\Phi\left(u_{n}+\varepsilon_{0}\right) \leq \Phi\left(u_{n}\right)+\frac{1}{n}
$$


By the compactness theorem, assume that $u_{n} \longrightarrow u_{0}$. If $u_{n} \searrow u_{0}$, since $\Phi$ is right continuous, we get $\Phi\left(u_{0}+\varepsilon_{0}\right) \leq$ $\Phi\left(u_{0}\right)$, a contradiction. If $u_{n} \nearrow u_{0}$, since $\lim _{u_{n} \rightarrow u_{0}} \Phi\left(u_{n}\right) \leq \Phi\left(u_{0}\right)$, we have

$$
\lim _{n \rightarrow \infty} \Phi\left(u_{n}\right)=b \leq \Phi\left(u_{0}\right)
$$

Moreover, $u_{n}+\varepsilon_{0}>u_{0}+\varepsilon_{0}$; we have known that there exists $n_{1} \in N, n>n_{1}$ such that $\Phi\left(u_{n}+\varepsilon_{0}\right)>\Phi\left(u_{0}\right)$, which gives $\lim _{n \rightarrow \infty} \Phi\left(u_{n}+\varepsilon_{0}\right)>\Phi\left(u_{0}\right)$. This shows that

$$
\Phi\left(u_{0}\right)<\lim _{n \rightarrow \infty} \Phi\left(u_{n}+\varepsilon_{0}\right) \leq \lim _{n \rightarrow \infty} \Phi\left(u_{n}\right)=b \leq \Phi\left(u_{0}\right),
$$

a contradiction.

Put $G_{n}(D)=\left\{t \in G: x_{n}(t) /\left\|x_{n}\right\|_{F} \geq D\right\}$ for each $n \in N$. From condition (44) there exists a $D>0$ such that $m\left(G_{n}\right.$ $(D)) \leq\left(\delta_{n} / 2\right)$. Put $G_{n_{1}}=G_{n} \backslash G_{n}(D)$ for each $n \in N$. Then,

$$
m\left(G_{n_{1}}\right) \geq m\left(G_{n}\right)-m\left(G_{n}(D)\right) \geq \frac{\delta_{0}}{2} .
$$

Therefore,

$$
\frac{x(t)}{\left\|x_{n}\right\|_{F}}-\frac{x_{n}(t)}{\left\|x_{n}\right\|_{F}} \geq \frac{\varepsilon_{0}}{\left\|x_{n}\right\|_{F}} \geq \frac{\varepsilon_{0}}{\|x\|_{F}}\left(t \in G_{n_{1}}\right) .
$$

Without loss of generality, we may assume that $\left(\|x\|_{F}\right.$ 12) $\leq\left\|x_{n}\right\|_{F}$ for each $n \in N$. Put $\varepsilon_{1}=2 \varepsilon_{0} /\|x\|_{F}$. Applying condition (46), there exists a $\delta_{1} \geq 0$ such that $\Phi\left(u+\varepsilon_{1}\right)$ $\geq \Phi(u)+\delta_{1}$ when $u \in[0, D]$.

Using $0 \leq x_{n}(t) \leq x(t)$ and $\left\|x_{n}\right\|_{F} \nearrow\|x\|_{F}$ as $n \longrightarrow \infty$, we have $\Phi\left(x(t) /\left\|x_{n}\right\|_{F}\right) \longrightarrow \Phi\left(x(t) /\left\|x_{n}\right\|_{F}\right)$ thanks to $\Phi$ that is right continuous. Then, there exists a constant $\alpha>0$ such that $\Phi\left(x(t) /\left\|x_{n}\right\|_{F}\right) \leq \Phi(\alpha x(t))$ for all $n \in N$ and $\mu$-a.e.t $\epsilon$ $\Omega$. Since $\Phi \in \Delta_{2}$, we have $\int_{G} \Phi(\alpha x(t)) d t<\infty$. By the Lebesgue dominated convergence theorem, we obtain

$$
\int_{G} \Phi\left(\frac{x(t)}{\left\|x_{n}\right\|_{F}}\right) d t \longrightarrow \int_{G} \Phi\left(\frac{x(t)}{\|x\|_{F}}\right) d t=\|x\|_{F}
$$

Consequently,

$$
\begin{aligned}
& \|x\|_{F} \longleftarrow \int_{G} \Phi\left(\frac{x(t)}{\left\|x_{n}\right\|_{F}}\right) d t \geq \int_{G_{n_{1}}} \Phi\left(\frac{x_{n}(t)+\varepsilon_{0}}{\left\|x_{n}\right\|_{F}}\right) \\
& \cdot d t+\int_{G \backslash G_{n_{1}}} \Phi\left(\frac{x(t)}{\left\|x_{n}\right\|_{F}}\right) d t \geq \int_{G_{n_{1}}} \Phi\left(\frac{x_{n}(t)}{\left\|x_{n}\right\|_{F}}+\frac{\varepsilon_{0}}{\|x\|_{F}}\right) \\
& \cdot d t+\int_{G \backslash G_{n_{1}}} \Phi\left(\frac{x(t)}{\left\|x_{n}\right\|_{F}}\right) d t \geq \int_{G_{n_{1}}} \Phi\left(\frac{x_{n}(t)}{\left\|x_{n}\right\|_{F}}\right)
\end{aligned}
$$

$$
\begin{aligned}
& \cdot d t+\int_{G_{n_{1}}} \delta_{1} d t+\int_{G \backslash G_{n_{1}}} \Phi\left(\frac{x(t)}{\left\|x_{n}\right\|_{F}}\right) \\
& \cdot d t=\int_{G_{n_{1}}} \Phi\left(\frac{x_{n}(t)}{\left\|x_{n}\right\|_{F}}\right) d t+\frac{\delta_{0} \delta_{1}}{2}+\int_{G \backslash G_{n_{1}}} \Phi\left(\frac{x(t)}{\left\|x_{n}\right\|_{F}}\right) \\
& \cdot d t \geq \int_{G_{n_{1}}} \Phi\left(\frac{x_{n}(t)}{\left\|x_{n}\right\|_{F}}\right) d t+\frac{\delta_{0} \delta_{1}}{2}+\int_{G \backslash G_{n_{1}}} \Phi\left(\frac{x_{n}(t)}{\left\|x_{n}\right\|_{F}}\right) \\
& \cdot d t=\int_{G} \Phi\left(\frac{x_{n}(t)}{\left\|x_{n}\right\|_{F}}\right) d t+\frac{\delta_{0} \delta_{1}}{2}=\left\|x_{n}\right\|_{F}+\frac{\delta_{0} \delta_{1}}{2},
\end{aligned}
$$

which contradicts with the assumption $\lim _{n \rightarrow \infty}\left\|x_{n}\right\|_{F}=\|x\|_{F}$. Therefore, $x_{n}$ is convergent to $x$ in measure.

Hence, $x_{n} /\left\|x_{n}\right\|_{F} \longrightarrow^{\mu} x /\|x\|_{F}$. By the fact that there is absolute continuity of integral, for any $\varepsilon>0$, there exists $\delta_{2}>0$ such that

$$
\int_{E_{0}} \Phi\left(\frac{x(t)}{\|x\|_{F}}\right) d t<\varepsilon
$$

for every measureable set $E \subset G$ with $m(E)<\delta_{2}$.

Consequently, this implies $\lim _{n \rightarrow \infty} \int_{E_{0}} \Phi\left(x_{n}(t) /\left\|x_{n}\right\|_{F}\right) d t=$ $\int_{E_{0}} \Phi\left(x(t) /\|x\|_{F}\right) d t$. By the Egorov theorem, for the above $\delta_{2}>0$, there exists $E_{0} \subset G$ such that $m\left(E_{0}\right)<\delta_{2}$ and

$$
\frac{x_{n}(t)}{\left\|x_{n}\right\|_{F}} \longrightarrow \frac{x(t)}{\|x\|_{F}} \text { uniformly in } \mathrm{t} \in G \backslash E_{0} .
$$

Since $\lim _{u \rightarrow 0+0} \Phi(u)=0$, we get

$$
\lim _{n \rightarrow \infty} \int_{G \backslash E_{0}} \Phi\left(\frac{x(t)}{\|x\|_{F}}-\frac{x_{n}(t)}{\left\|x_{n}\right\|_{F}}\right) d t=0 .
$$

Consequently,

$$
\begin{gathered}
\int_{G} \Phi\left(\frac{x(t)}{\|x\|_{F}}-\frac{x_{n}(t)}{\left\|x_{n}\right\|_{F}}\right) d t=\int_{G \backslash E_{0}} \Phi\left(\frac{x(t)}{\|x\|_{F}}-\frac{x_{n}(t)}{\left\|x_{n}\right\|_{F}}\right) \\
\cdot d t+\int_{E_{0}} \Phi\left(\frac{x(t)}{\|x\|_{F}}-\frac{x_{n}(t)}{\left\|x_{n}\right\|_{F}}\right) d t \leq \int_{G \backslash E_{0}} \Phi\left(\frac{x(t)}{\|x\|_{F}}-\frac{x_{n}(t)}{\left\|x_{n}\right\|_{F}}\right) \\
\cdot d t+\int_{E_{0}} \Phi\left(\frac{x(t)}{\|x\|_{F}}\right) d t \leq \varepsilon+\varepsilon=2 \varepsilon .
\end{gathered}
$$

By the arbitrariness of $\varepsilon$, we obtain that $\left\|x-x_{n}\right\|_{E} \longrightarrow 0$ as $n \longrightarrow \infty$ holds

In virtue of Theorem 14, we have a necessity that is obvious.

Theorem 16. For any $x \in l_{\Phi} \backslash\{0\}, I_{\Phi}\left(x /\|x\|_{F}\right)=\|x\|_{F}$ if and only if

(i) $\Phi \in \delta_{2}$

(ii) $\Phi$ is right continuous 
Proof. Necessity:

(i) If $\Phi \notin \delta_{2}$, then there exists an element $x \in l_{\Phi}$ such that $I_{\Phi}(x)<1$ and $I_{\Phi}(x / \lambda)=\infty$ for any $0<\lambda<1$. Hence, $\|x\|_{F}=1$ and $I_{\Phi}\left(x /\|x\|_{F}\right)<\|x\|_{F}$

(ii) If there exists a point $u_{0} \in(0,+\infty)$ such that $\Phi\left(u_{0}\right)$ $<\lim _{u \rightarrow u_{0}+0} \Phi(u)=b$, that is, for any $0<\lambda<1$, we get $\Phi\left(u_{0} / \lambda\right) \geq b$

Put $x=\left(b u_{0}, 0, \cdots\right)$. Then, $I_{\Phi}(x / b)=\Phi\left(u_{0}\right)<b$ and for any $0<\lambda<1$, we have $I_{\Phi}(x / \lambda b)=\Phi\left(b u_{0} / \lambda b\right) \geq b$. Hence, $\| x$ $\|_{F}=b$ and $I_{\Phi}\left(x /\|x\|_{F}\right)<\|x\|_{F}$.

The sufficiency of the proof is similar as Theorem 11.

Theorem 17. The following statements are equivalent:

(1) The Orlicz sequence space $l_{\Phi}$ equipped with the Mazur-Orlicz F-norm is lower locally uniformly monotone

(2) The Orlicz sequence space $l_{\Phi}$ equipped with the Mazur-Orlicz F-norm is strictly monotone

(3) (a) $\Phi$ is strictly increasing on $R^{+}$

(b) $\Phi \in \delta_{2}$

(c) $\Phi$ is right continuous

Proof. The implication $(1) \Longrightarrow(2)$ is clear.

(2) $\Longrightarrow(3)$ The proof is similar as the proof of Theorem 15. We only give the proof that $\Phi$ is right continuous, if there exists a point $u_{0} \in(0,+\infty)$ such that $\Phi\left(u_{0}\right)<\lim _{u \rightarrow u_{0}+0} \Phi(u)=b$. That is, for any $0<\lambda<1$, we get $\Phi\left(u_{0} / \lambda\right) \geq b$. Put $x=\left(b u_{0}\right.$, $0, \cdots)$. Then, $I_{\Phi}\left(x /\|x\|_{F}\right)<\|x\|_{F}$, Since $\lim _{u \rightarrow 0+0} \Phi(u)=0$, there exists a $c>0$ such that $\Phi\left(b u_{0} / b\right)+\Phi(c / b) \leq\|x\|_{F}=b$. Put $y=$ $\left(b u_{0}, c, 0, \cdots\right)$. Then, $b=\|x\|_{F} \leq\|y\|_{F} \leq b, 0 \leq x \leq y$ and $x \neq y$.

(3) $\Longrightarrow(1)$ The idea is the same as the proof of Theorem 15 , and the proof is simpler than Theorem 15 . We only need to replace convergence by the measure of convergence by coordinate.

\section{Data Availability}

No data were used to support this study.

\section{Conflicts of Interest}

The authors declare that there is no conflict of interest regarding the publication of this paper.

\section{Acknowledgments}

This work is supported by the National Natural Science Foundation of China under Grant no. 11871181.

\section{References}

[1] S. Chen, Y. Cui, and H. Hudzik, "Isometric copies of $l^{1}$ and $l^{\infty}$ in Orlicz spaces equipped with the Orlicz norm," Proceedings of the American Mathematical Society, vol. 132, no. 2, pp. 473-481, 2004.

[2] Y. Cui, H. Hudzik, L. Szymaszkiewicz, and T. Wang, "Criteria for monotonicity properties of Musielak-Orlicz spaces equipped with the Amemiya norm," Journal of Mathematical Analysis and Applications, vol. 303, no. 2, pp. 376-390, 2005.

[3] M. Ciesielski, P. Kolwicz, and R. Płuciennik, "Local approach to Kadec-Klee properties in symmetric function spaces," Journal of Mathematical Analysis and Applications, vol. 426, no. 2, pp. 700-726, 2015.

[4] F. Albiac and J. L. Ansorena, "Integration in quasi-Banach spaces and the fundamental theorem of calculus," Journal of Functional Analysis, vol. 264, no. 9, pp. 2059-2076, 2013.

[5] W. Kurc, "Strictly and uniformly monotone Musielak-Orlicz spaces and applications to best approximation," Journal of Approximation Theory, vol. 69, no. 2, pp. 173-187, 1992.

[6] Y. Cui, H. Hudzik, R. Kaczmarek, and P. Kolwicz, "Geometric properties of F-normed Orlicz spaces," Aequationes Mathematicae, vol. 93, no. 1, pp. 311-343, 2019.

[7] H. Hudzik, A. Kamińska, and M. Mastyło, "Monotonicity and rotundity properties in Banach lattices," Rocky Mountain Journal of Mathematics, vol. 30, no. 3, pp. 933-950, 2000.

[8] T. Dominguez, H. Hudzik, G. López, M. Mastyło, and B. Sims, "Complete characterization of Kadec-Klee properties in Orlicz spaces," Houston Journal of Mathematics, vol. 29, no. 4, pp. 1027-1044, 2003.

[9] S. T. Chen, X. He, and H. Hudzik, "Monotonicity and best approximation in Banach lattices," Acta Mathematica Sinica, vol. 25, no. 5, pp. 785-794, 2009.

[10] M. Ciesielski, P. Kolwicz, and A. Panfil, "Local monotonicity structure of symmetric spaces with applications," Journal of Mathematical Analysis and Applications, vol. 409, no. 2, pp. 649-662, 2014.

[11] P. Kolwicz, "Local structure of symmetrizations $E^{*}$ with applications," Journal of Mathematical Analysis and Applications, vol. 440, no. 2, pp. 810-822, 2016.

[12] B. Bru and H. Heinich, "Monotonies des espaces d'Orlicz," Comptes rendus de l'Académie des sciences. Série 1, Mathématique, vol. 301, no. 19, pp. 893-894, 1985.

[13] H. Hudzik, X. B. Liu, and T. F. Wang, "Points of monotonicity in Musielak-Orlicz function spaces endowed with the Luxemburg norm," Archiv der Mathematik, vol. 82, no. 6, pp. 534545,2004

[14] N. J. Kalton, N. T. Peck, and J. W. Roberts, An F-Space Sampler, Cambridge University Press, 1984.

[15] C. Shutao, Geometry of Orlicz Spaces, Institute of Mathematics, Polish Academy of Science, Warszawa, 1996. 